\title{
Incubation in Cyclohexene Decomposition at High Temperatures
}

\author{
JICHUN SHI and JOHN R. BARKER \\ Department of Atmospheric, Oceanic and Space Sciences, Space Physics Research Laboratory, \\ The University of Michigan, Ann Arbor, Michigan 48109-2143
}

\begin{abstract}
A detailed master equation simulation has been carried out for the thermal unimolecular decomposition of $\mathrm{C}_{6} \mathrm{H}_{10}$ in a shock tube. At the highest temperatures studied experimentally [J.H. Kiefer and J. N. Shah, J. Phys. Chem., 91, 3024 (1987)], the average thermal vibrational energy is greater than the reaction threshold and therefore $\langle\Delta E\rangle$ (up and down steps) is positive for molecules at that energy, rather than negative; the converse is true at lower temperatures. The calculated incubation time, in which the decomposition rate constant rises to $1 / \mathrm{e}$ of its steady state value, is found to be only weakly dependent on temperature (at constant pressure i between $1500 \mathrm{~K}$ and $2000 \mathrm{~K}$ and to depend almost exclusively on $\langle\Delta E\rangle_{d}$ (down steps, only), and not on collision probability model. Simulations of the experimental data show the magnitude of $\langle\Delta E\rangle_{d}$ depends weakly on assumed collision probability model, but is nearly independent of temperature. The second moment $\langle\Delta E\rangle^{1 / 2}$ is found to be independent of both temperature and transition probability model. The experimental data are not very sensitive to the possible energy-dependence of $\langle\Delta E\rangle_{d}$ for a wide range of assumptions. It is concluded that the observed experimental "delay times" probably can be identified with the incubation time; further experiments are desirable to test this possibility and obtain more direct measures of the incubation time.
\end{abstract}

\section{Introduction}

In a shocked gas, the translational temperature increases very rapidly due to adiabatic compression, and the rotational energy keeps pace, because translational-rotational (T-R) energy transfer is very efficient. In contrast, translational-vibrational (T-V) energy transfer is relatively inefficient and the vibrational energy content of the molecules in the gas lags well behind that of the other degrees of freedom [1]. The unimolecular reaction rate depends primarily on vibrational energy, and therefore unimolecular reactions exhibit a delay while the vibrational energy distribution is relaxing to the higher kinetic temperature of the shock. This delay is called the incubation time, and it depends on the energy transfer properties of the reactant and collider gases, as well as the nature of the chemical reaction.

Dove, et al. [2] studied the incubation of $\mathrm{N}_{2} \mathrm{O}$ and found an incubation time of about 4-7 times the relaxation time for the temperatures of 2000$3500 \mathrm{~K}$. Pritchard ${ }^{3}$ also has investigated the incubation process in unimolecular reaction systems. Incubation has also been studied by the variable encounter method (VEM) [4], but this technique only applies to heterogeneous collisional activation. 
Conventional steady-state unimolecular rate theory for thermal reactions is not appropriate for descriptions of incubation, because the population distributions are nonthermal and time-dependent, hence not at steady-state. Instead, master equation calculations are required, where energy transfer models and the specific unimolecular rate constants $(k(E)$ 's) are used explicitly [5]. Dove and Troe [6] carried out such an analysis for the decomposition of $\mathrm{N}_{2} \mathrm{O}$ and showed that the incubation time depends critically on the energy transfer properties of the system, as expected. Malins and Tardy [7] analyzed the incubation process in cyclopropane, using an energy-grained master equation (175 levels with a spacing of about $350 \mathrm{~cm}^{-1}$ ), and the calculated incubation time was found to be short, compared to the reaction time constant.

In small molecules, even at several thousand degrees, the average thermal vibrational energy is usually much lower than the critical energy $E_{0}$ for reaction and, at thermal equilibrium, only the high energy tail of the distribution function participates in the reaction. Under these conditions, molecules with sufficient energy to react tend to be deactivated in collisions and the average energy transfer in a collision is negative: $\left\langle\Delta E\left(E_{0}\right)\right\rangle<$ 0 . In large molecules at moderate temperatures, however, the average thermal energy can be significantly higher than the reaction threshold, and thus for molecules near the threshold, $\left\langle\Delta E\left(E_{0}\right)\right\rangle>0$. Because of this characteristic of large molecules, Tzidoni and Oref [8] have questioned whether large molecules can ever reach steady state in shocked systems (i.e., infinite incubation time). In any event, the incubation time in such systems is likely to be different from that of small molecules.

Recently, Kiefer and Shah [9] (KS) used a shock tube to investigate the decomposition of cyclohexene to produce ethylene and 1,3-butadiene:

$$
c-\mathrm{C}_{6} \mathrm{H}_{10} \longrightarrow \mathrm{C}_{2} \mathrm{H}_{4}+1,3-\mathrm{C}_{4} \mathrm{H}_{6}
$$

This reverse Diels-Alder reaction produces only molecular products and is very well-behaved kinetically. They investigated the reaction in the temperature range from $1200-2000 \mathrm{~K}$, and other groups $[10,11]$ have investigated the same reaction from $814-1490 \mathrm{~K}$. The pressure range investigated by KS was limited (ca. 100 to 500 torr), but it clearly showed fall-off of the nimolecular rate constant. Of particular interest for present purposes, they achieved very high temperatures and used the laser schlieren (LS) and pulsed laser flash absorption (PLFA) techniques, with time resolutions $(<0.1 \mu \mathrm{s})$ potentially capable of observing the incubation time.

In principle, the fast time resolution in the KS experiments would permit the investigation of incubation times. However, there are complications due to uncertainties in the knowledge of the exact time of passage of the shockfront (time origin), and laser beam/shock-front interactions tend to dominate the signal just when incubation is occuring [9]. Despite these problems, the data may provide some information about the incubation process.

In the present article, we have used a full collision/reaction Master Equation to simulate the decomposition of cyclohexene under the conditions of the KS experiments. Unlike the calculations carried out by Malins and Tardy [7], energy-graining was not used, permitting better numerical simulations of small collisional step sizes. One objective of this work was to 
determine whether steady-state is achieved under the conditions of the experiments, and whether incubation time measurements can provide more information about the energy transfer properties of the system. Such properties include energy transfer transition probability distributions, the energy-dependence of $\langle\Delta E\rangle$, which was recently confirmed to be approximately linear for azulene [12], and the temperature dependence of $\langle\Delta E\rangle_{d}$, which has been shown to be small for other systems [13]. A third objective was to determine whether Master Equation calculations can be used in conjunction with the experiments to determine whether the "delay" observed in the experimental studies on this system is due to incubation.

In the following section, the LS technique is described, in order to show how the "time origin" uncertainty and laser beam/shock-front interactions affect the determination of incubation time. Then, the Master Equation simulation is described briefly, including the RRKM rate constants and collisional transition probability models used in the calculations. After a section reporting the results and discussion, the conclusions are presented.

\section{Background Information}

\section{Shock Tube Laser Schlieren Technique}

The shock tube laser schlieren technique has been described in detail in the literature [14]. Briefly, a shock wave adiabatically compresses the gas and raises its temperature from room temperature to as much as several thousand degrees, almost instantaneously. The reactant molecules approach the new thermodynamic equilibrium by a series of collisions with the collider gas molecules. Decomposition of the reactant begins after the short delay necessary to excite the reactant above the reaction critical energy, as discussed below. The heat released from the chemical reactions produces density changes in the gas medium, which cause changes in the index of refraction.

Refraction of a small laser beam (about $0.3 \mathrm{~mm}$ in diameter) traversing the shock tube is used as the probe to monitor the index of refraction changes (and hence, the gas density gradient). The laser intensity is monitored by photoelectric techniques and a typical signal obtained from a differential detector is shown in Figure 1. The large disturbance near $t=0$ is caused by the interaction between the shock front and the laser beam, while the later signal is from chemical reaction. As pointed out by $\mathrm{KS}[9,15]$, extrapolation and interpolation of the later signal can give, with reasonable accuracy, the initial density gradient due to chemical reaction alone. It has been shown that the rate of the chemical process is proportional to the density gradient in the system. The initial rate constant is based on the initial density gradient and the enthalpy change $\Delta H_{R}$ for the chemical reaction. If one has a single reaction with known $\Delta H_{R}$, this technique should be able to determine the rate constant to better than $\pm 10 \%$.

The possible sources of error in the preliminary initial rate constants obtained by the method described above are the uncertainty in the extrapolated gradient and, in high temperature cases, the error in locating the "starting point" of the chemical reaction, which depends on the determina- 




Figure 1. Typical Signal for a shock tube laser schlieren measurement. The horizontal axis is time in $\mu \mathrm{s}$. (adapted from ref. [17]).

tions of both the "time origin" and the incubation time. As will be discussed later in this section, the determinations of both quantities are by no means free of uncertainties. Nevertheless, KS [9] concluded that these uncertainties have negligible effects on the derived rates, especially at lower temperature, because the extrapolation is very short.

There are several reports on the determination of the "time origin" in schlieren-shock tube systems. Dove and Teitlebaum [16] located the time origin at the first minimum in the schlieren signal (refer to Fig. 1). After detailed theoretical calculations, Kiefer et al. [17] concluded that the "time origin" is located about $0.2 \mu$ s later than the first minimum. A series of calculations using this theory for different temperatures suggests that the position of the starting point does not depend strongly on the temperature of the system. However, deviations from this theory have also been observed [18].

It should be emphasized that the "time origin" corresponds to the time when the shock front passes through the center of the laser beam. This can be taken as the moment when the temperature of the system is raised instantaneously. The time difference between the "time origin" and "starting point" for reaction is the incubation time, during which time the reactant molecules relax from the low internal energy nonequilibrium state at the "time origin" to energies high enough for reaction to occur. In large molecule systems with high temperature, the incubation time is likely to be significant. It was found by $\mathrm{KS}$ [9] in their $\mathrm{C}_{6} \mathrm{H}_{10}$ study that a "delay" in reaction of about $0.2 \mu \mathrm{s}$ had to be assumed to obtain a good fit to the experi- 
mental signal.As is discussed later in this article, it is likely that this "delay" corresponds to the incubation time. However, uncertainties in the "time origin" may also contribute to the observed "delay."

\section{Master Equation Simulations}

The Master equation implementation used here has been discussed elsewhere in detail [5]. Briefly, random numbers are used to select both the initial conditions and the progress variables for the stochastic simulation of each collision. Exact-count densities of states, based on the KS vibrational assignment for cyclohexene [9], were calculated using the SteinRabinovitch algorithm [19]. This Master Equation implementation obeys microscopic reversibility and detailed balance in calculating collisional energy transfer probabilities according to empirical models, which are described below. In the simulations, the specific unimolecular rate constants, $k(E)$, are calculated by the Inverse Laplace Transform (ILT) method [20] using the $E_{x}$ and $A_{x}$ data determined by KS. A direct comparison showed that the $k(E)$ values from the ILT method, based on $A_{\infty}$ and $E_{x}$ data from $\mathrm{KS}$, agreed very well with those calculated using the RRKM model which KS used to fit their data (Note: two of the cyclohexene transition state vibrational frequencies were listed incorrectly in KS [21]). When the population distribution has reached steady-state in the simulations, the timedependent reaction yield predicted by the Master Equation calculation is fitted by least squares to determine the steady-state rate constant; steady state was achieved in all of the calculations.

\section{Decomposition Rate Constant for $\mathrm{c}-\mathrm{C}_{6} \mathrm{H}_{10}$}

The thermal decomposition of $c-\mathrm{C}_{6} \mathrm{H}_{10}$ has been investigated in several shock tube experiments [9-11]. Secondary reactions of the reaction products are found to be negligible for $\mathrm{T}<2000 \mathrm{~K}$. Barnard and Parrott [10] investigated the reaction at $1010-1330 \mathrm{~K}$, including a study of the rate constant pressure fall-off, which had been neglected by prior workers. In order to determine the high pressure rate constant parameters, they used Forst's method [22] to study the fall-off, and their results give $\log \left(A_{x} / \mathrm{s}^{-1}\right)=15.18$ and $E_{x}=22912 \mathrm{~cm}^{-1}$. KS [9] studied the reaction at lower pressures and at higher temperatures than Barnard and Parrott, and their RRKM fit of the observed rate constants gave $\log \left(A_{\infty} / \mathrm{s}^{-1}\right)=15.57$ and $E_{\infty}=22990 \mathrm{~cm}^{-1}$. It should be noted that, in KS's RRKM calculation for this system, they employed Troe's original formulation [23] for energy transfer collision efficiency, but this formulation does not allow $\langle\Delta E\rangle$ to change sign at higher temperatures [13a]. This problem has been addressed in more detail in a recent study of this system by Tardy and Rabinovitch [24].

The experimental low pressure rate constants are required for comparison with the simulations. The $c-\mathrm{C}_{6} \mathrm{H}_{10}$ experimental rate constants were grouped together into two pressure ranges by KS: $360-550$ torr and 107-170 torr. But KS indicated that these groups of data have small scatter (averaging less than $5 \%$ ) and accurate least squares fits of the rate constants for each group as a function of temperature were obtained. 


\section{Collisional Transition Probability Models}

In each collision, the energy of both collisional partners will change, and both activation and deactivation are possible. The energy change of the reactant molecule can be described using empirical models for the collision step size distribution function $P_{i}\left(E, E^{\prime}\right)$. This function describes the probability that a molecule initially possessing internal energy $E$ will be found in the energy range $E^{\prime}$ to $E^{\prime}+d E$ following a single collision. The product $\omega_{i} P_{i}\left(E, E^{\prime}\right)$ is the effective first-order rate constant for populating energy $E^{\prime}$ from molecules initially at energy $E$. Here $\omega_{i}$ is the collisional frequency for the $i^{\text {th }}$ pair of distinguishable species and it is taken as the LennardJones collision frequency (Lennard-Jones parameters are taken from KS).

We have used three empirical transition probability models for deactivation collisions:

Exponential Model:

$$
P_{d}\left(E, E^{\prime}\right)=\exp \left(-\frac{E-E^{\prime}}{\alpha}\right) \quad E^{\prime}<E
$$

Reverse Exponential Model:

$$
P_{d}\left(E, E^{\prime}\right)=\exp \left(-3+\frac{2\left(E-E^{\prime}\right)}{\alpha}\right) \quad E \geq E^{\prime} \geq\left(E-\frac{3}{2} \alpha\right)
$$

Box Model:

$$
P_{d}\left(E, E^{\prime}\right)=c \quad E \geq E^{\prime} \geq(E-\alpha)
$$

where $E$ and $E^{\prime}$ are the energies before and after a collision, respectively, and $c$ in the Box model expression is a constant. For the convenience of normalization, $c$ is assumed to be 1 . The parameter $\alpha$ can be related to $\langle\Delta E\rangle_{d}$, the average energy transferred in downward transitions. The upward transition probability function $P_{u}\left(E, E^{\prime}\right)$ is calculated from $P_{d}\left(E^{\prime}, E\right)$ by assuming detailed balance:

$$
P_{u}\left(E, E^{\prime}\right)=P_{d}\left(E^{\prime}, E\right) \frac{\rho\left(E^{\prime}\right)}{\rho(E)} \exp \left[-\frac{\left(E^{\prime}-E\right)}{k T}\right] \quad E^{\prime}>E
$$

Here, $E^{\prime}$ is the energy after the collisional activation, and $\rho(E)$ is the density of states at energy $E$. The total upward and downward transitional probabilities can be calculated from $P_{d}\left(E, E^{\prime}\right)$ and the normalization condition must be satisfied:

$$
\int_{0}^{E} \frac{1}{N(E)} P_{d}\left(E, E^{\prime}\right) d E^{\prime}+\int_{E}^{\infty} \frac{1}{N\left(E^{\prime}\right)} P_{u}\left(E, E^{\prime}\right) d E^{\prime}=1
$$

$N(E)$ and $N\left(E^{\prime}\right)$ are the normalization factors for the proposed microcannonical transition probability function at energy $E$ and $E^{\prime}$, respectively, and are not equal to each other, in general.

In this article, two methods were used to evaluate the normalization factor: the "bootstrap" method described by Barker [5] and the finite difference method described by Gilbert and King [25]. In Barker's method, $N(E)$ and $N\left(E^{\prime}\right)$ are assumed to be equal to each other at high energy; then the 
energy-dependent normalization is evaluated at lower energies, based on those at higher energies coupled with an extrapolation technique. In all of the numerical calculations, a $25 \mathrm{~cm}^{-1}$ energy increment was used. The two methods agree very well for $E>10,000 \mathrm{~cm}^{-1}$, and at lower energies, the difference between the two methods never exceeds $15 \%$. This error arises mostly because the density of states in the "bootstrap" method is obtained from logarithmic interpolation of exact count densities of states that were obtained in a separate calculation. This interpolation error of ca. $15 \%$ for $E<10,000 \mathrm{~cm}^{-1}$ affects the calculated incubation times by less than $10 \%$, because $\rho(E)$ increases very rapidly in the low energy region. Because the sacrifice in accuracy is not large, and because the bootstrap method is more efficient numerically, it was used in all calculations reported here.

Normalized step size transition probability distributions for the three models are presented in Figure 2. The Exponential and Reverse Exponential models were used in the simulations of azulene energy transfer studies (Ref. [12(a)]) carried out in this laboratory. The Box model is new here, and

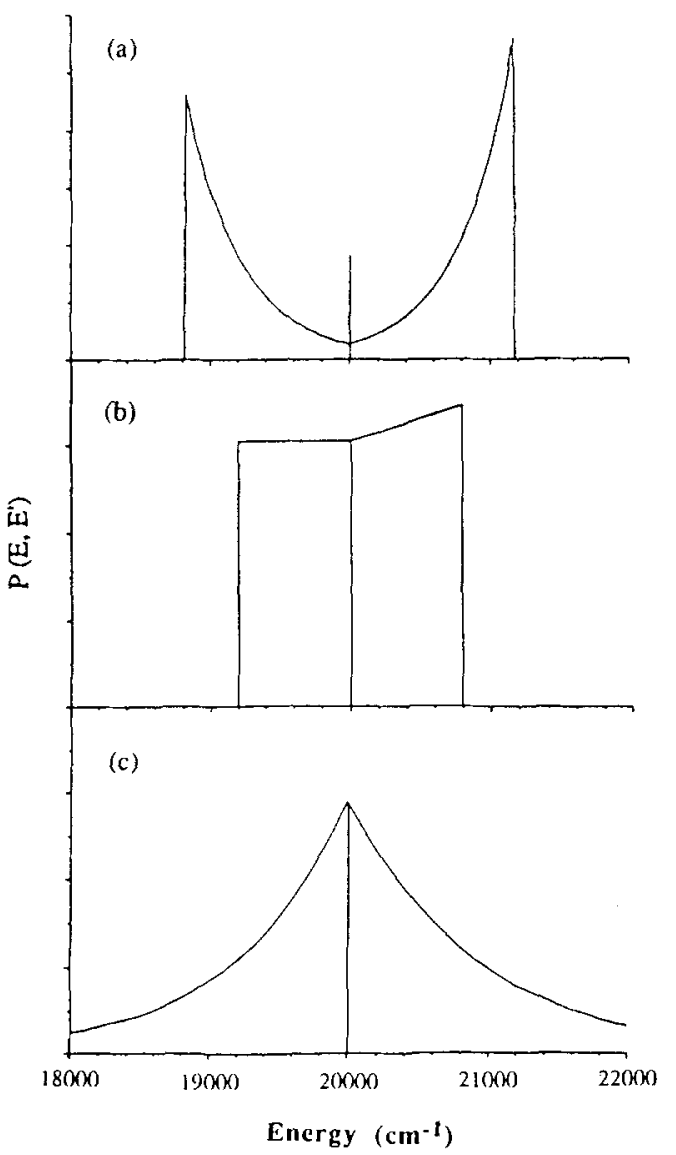

Figure 2. Normalized transition probability functions $P\left(E, E^{\prime}\right)$ for the three empirical functions, with $\langle\Delta E\rangle_{d}=800 \mathrm{~cm}^{-1}$ and $T=1750 \mathrm{~K}$. (a) Reverse exponential model; (b) Box model; and (c) Exponential model. 
is motivated by the finding of Nguyen et al. [26] in their multichannel pyrolysis study on bromoethane that the "exponential-cube model" fitted their experimental data better than the exponential model. The Box model is the limiting case of the generalized Gaussian models described by eq. (7):

$$
P_{d}\left(E, E^{\prime}\right)=\exp \left[-\left(\frac{E-E^{\prime}}{\alpha}\right)^{n}\right] \quad E>E^{\prime}
$$

The "exponential-cube model" corresponds to $n=3$ and the Box Model corresponds to $n=\infty$. The three empirical models used in the present work are intended to span a wide range of shapes, as shown in Figure 2, but other models can be developed that would fit the experimental data and the examples used here are not unique.

Properties of the transition probability distribution, such as $\langle\Delta E\rangle_{d},\langle\Delta E\rangle$ and $\left\langle\Delta E^{2}\right\rangle$, can be calculated using the usual formulae:

$$
\begin{aligned}
\langle\Delta E(E)\rangle_{d} & =\frac{\int_{0}^{E}\left(E^{\prime}-E\right) P_{d}\left(E, E^{\prime}\right) d E^{\prime}}{\int_{0}^{E} P_{d}\left(E, E^{\prime}\right) d E^{\prime}} \\
\langle\Delta E(E)\rangle & =\frac{\int_{0}^{\infty}\left(E^{\prime}-E\right) P\left(E, E^{\prime}\right) d E^{\prime}}{\int_{0}^{\infty} P\left(E, E^{\prime}\right) d E^{\prime}} \\
\left\langle\Delta E^{2}(E)\right\rangle & =\frac{\int_{0}^{\infty}\left(E^{\prime}-E\right)^{2} P\left(E, E^{\prime}\right) d E^{\prime}}{\int_{0}^{\infty} P\left(E, E^{\prime}\right) d E^{\prime}}
\end{aligned}
$$

where $P\left(E, E^{\prime}\right)$ is the total transition probability for both the upward and the downward transitions and $E^{\prime}$ is the energy after a collision. [Note that Gilbert [27] has argued that $\langle\Delta E\rangle_{d}$ is preferred over $\langle\Delta E\rangle$ in describing energy transfer, because the former quantity removes the trivial temperature-dependence imposed by detailed balance.]

In recent years, several physical methods have been used to obtain energy transfer properties from experimental observables, including time-resolved infrared emission [28], time-dependent thermal lensing [29], time-resolved optoacoustics [30], and ultraviolet absorption [31]. Often, $\langle\Delta E\rangle$ can be determined more or less directly, but this quantity provides little information concerning the most appropriate energy transfer model. Other quantities, such as $\left\langle\Delta E^{2}\right\rangle$, may be more useful for low pressure unimolecular reaction rate constants than $\langle\Delta E\rangle$ alone [32]. The weak-collision efficiency factor $\beta_{c}$ can be related directly to $\left\langle\Delta E^{2}\right\rangle$ by the following expressions, respectively $[33,34]$ :

$$
\beta_{c}=\frac{\left\langle\left\langle\Delta E^{2}\right\rangle\right\rangle_{s s}}{2(k T)^{2}} \text { and } \frac{\left\langle\Delta E^{2}\right\rangle}{2\left(F_{E} k T\right)^{2}}
$$


Here $\left\langle\left\langle\Delta E^{2}\right\rangle\right\rangle_{s s}$ is the steady-state bulk average of $\left\langle\Delta E^{2}\right\rangle$. It should be emphasized that these relations are independent of the transition probability model.

By simulating the thermal decomposition of cyclohexene in the shock tube using detailed master equation calculations, the $\langle\Delta E\rangle_{d}$ values for the given reaction conditions can be obtained by matching the calculated to the experimental rate constants, for any chosen transition probability model. After such parameter fitting, the master equation simulations give an accurate empirical description of the incubation process and self-consistent incubation times can be determined.

\section{Results and Discussion}

Master equation simulations as described above have been performed for five temperatures using all three transition probability models. At the lower temperatures, the average thermal energy $\langle E\rangle_{T}$ of $c-\mathrm{C}_{6} \mathrm{H}_{10}$ was lower than the reaction critical energy $E_{0}$, but at the higher temperatures, $\langle E\rangle_{T}$ is greater than $E_{0}$. Thus, at lower temperatures, molecules with energy $E_{0}$ will tend to be deactivated by collisions, while the reverse is true at the higher temperatures. The experimental rate constants from KS are grouped into two pressure ranges (107-170 torr and 360-550 torr). The rate constants in the lower pressure group are preferred for the calculations, because the reactions are in the intermediate fall-off region (refer to Fig. 5) and energy transfer is more important at lower pressures. In most calculations, the pressure was fixed at 150 torr, which falls in the middle of the lower pressure group of experiments.

Reaction yields $Y(t)$ were calculated as a function of time and an example is presented in Figure 3 along with $\ln (1-Y)$. The solid line is the least squares fit of the calculated values after the system has reached steady-state; the slope of the linear portion of $\ln (1-Y)$ vs. time gives the steady-state unimolecular rate constant $k_{\text {uni }}(T)$. Tzidoni and Oref [8] found that at high temperatures and low pressures, steady-state may not be achieved before the reaction is essentially complete, when approached from an initial high-temperature thermal distribution. In all of our calculations, steady-state was approached from an initial low-temperature thermal distribution and steady-state was achieved in all cases before the reaction yield exceeded $15 \%$.

At each temperature and for each model, "standard" simulations were arried out by varying $\langle\Delta E\rangle_{d}$ until the calculated steady-state rate constant agreed with the experimental value from KS. These "standard" simulations are for a total pressure of 150 torr and a dilution ratio of $10^{-5}$ of cyclohexene in $\mathrm{Kr}$; the resulting values of $\langle\Delta E\rangle_{d}$ are shown in Figure 4. (In the experiments, the dilution ratios were 0.02 and 0.04 and the reaction exothermicity significantly affected the bath temperature; this complication was avoided in the simulations by using a very small dilution ratio.) For each collision transition model, the corresponding $\langle\Delta E\rangle_{d}$ required to simulate the steady-state decomposition rate constant does not depend 


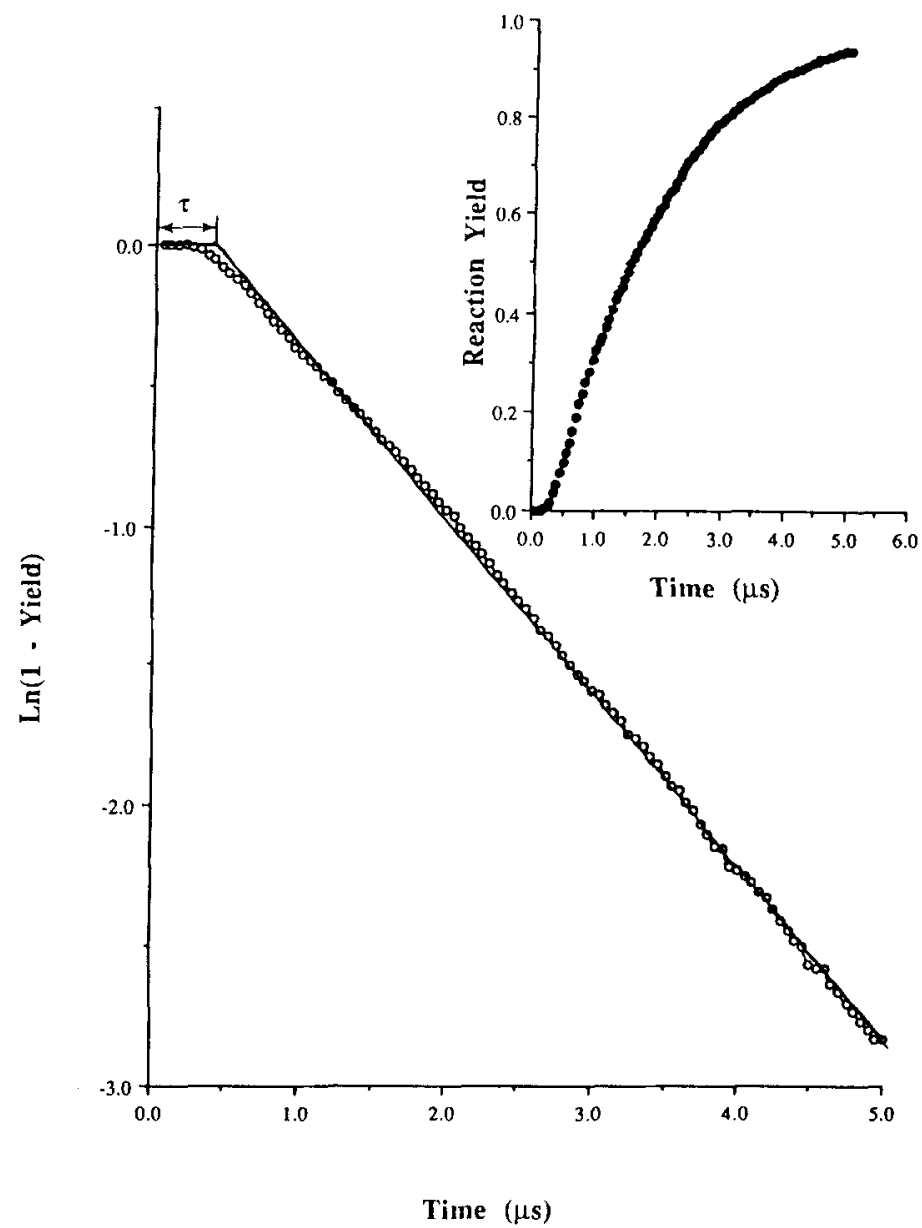

Figure 3. Typical example (exponential model) of the $\operatorname{Ln}(1-Y)$ vs. time curve, where $P=150$ torr, $T=1750 \mathrm{~K}$. The solid line is the least square fit of the calculated points. The short delay $\tau$ is the incubation time. The insert is the time dependent reaction yield under the same conditions.

strongly on temperature between $1500-2000 \mathrm{~K}$, in general agreement with some experiments on other molecules $[13,35]$. Note that the magnitude of $\langle\Delta E\rangle_{d}$ depends to some extent on the transition probability models, making determination of $\langle\Delta E\rangle_{d}$ from experimental macroscopic observables modeldependent.

The effects of fall-off were investigated by running simulations for pressures from 1 to 8000 torr at $1750 \mathrm{~K}$, using the Exponential transition probability model with the $\langle\Delta E\rangle_{d}$ from standard simulations, and the resulting unimolecular rate constants are presented in Figure 5 . As is clearly demonstrated in the figure, we can not simulate both the high and low pressure experimental rate constants with the same energy transfer parameters, indicating an inconsistency with the experimental study. This has also been noted in the Tardy and Rabinovitch study [24] on this reac- 


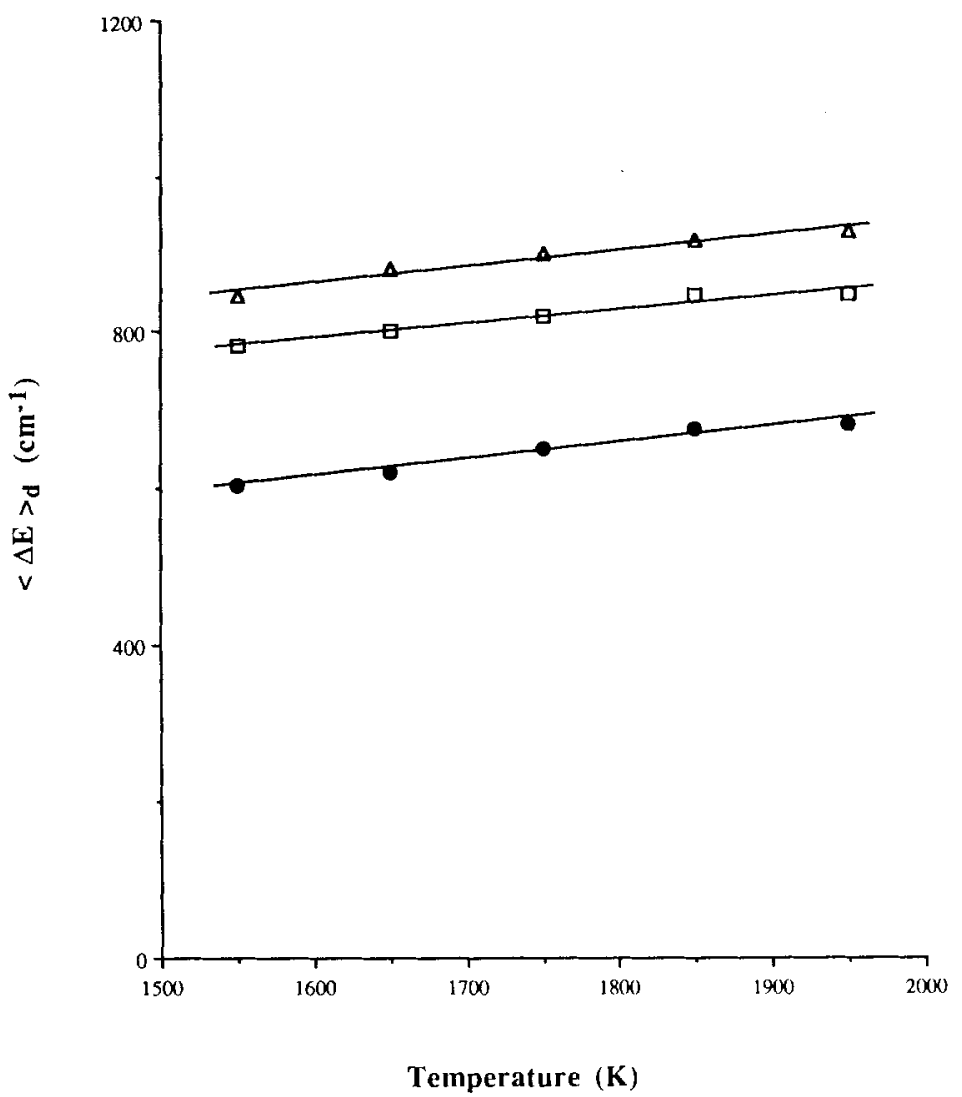

Figure 4. $\langle\Delta E\rangle_{d}$ 's used in the simulations. Solid circle: exponential model; Triangle: reverse exponential model; and Square: box model.

tion. Inspection of the figure indicates that the KS experiments at 150 torr are near the low pressure limit, but they are affected significantly by falloff. At high pressures, the cyclohexene population distribution should approach a canonical distribution, while at low pressures, the steady-state distribution should exhibit the characteristic depletion of molecules above the reaction threshold. These effects are reproduced in the Master Equation simulations, as illustrated in Figure 6.

It is apparent from Figure 3 that the decomposition of $c-\mathrm{C}_{6} \mathrm{H}_{10}$ is delayed for a short time under the conditions assumed. This was found to be the case in every simulation carried out in the present work, and the delay can be identified with the incubation time $\tau$. The incubation time can be defined quantitatively by the following expression for the yield in the steadystate region:

$$
Y=\left\{1-\exp \left[-k_{\text {uni }}(t-\tau)\right]\right\}
$$

where $k_{\text {uni }}$ is the steady-state unimolecular rate constant. The incubation time $\tau$ corresponds to the time when the straight-line portion of a $\ln (1-Y)$ vs. time plot passes through zero (refer to Fig. 3); it also corresponds to the 


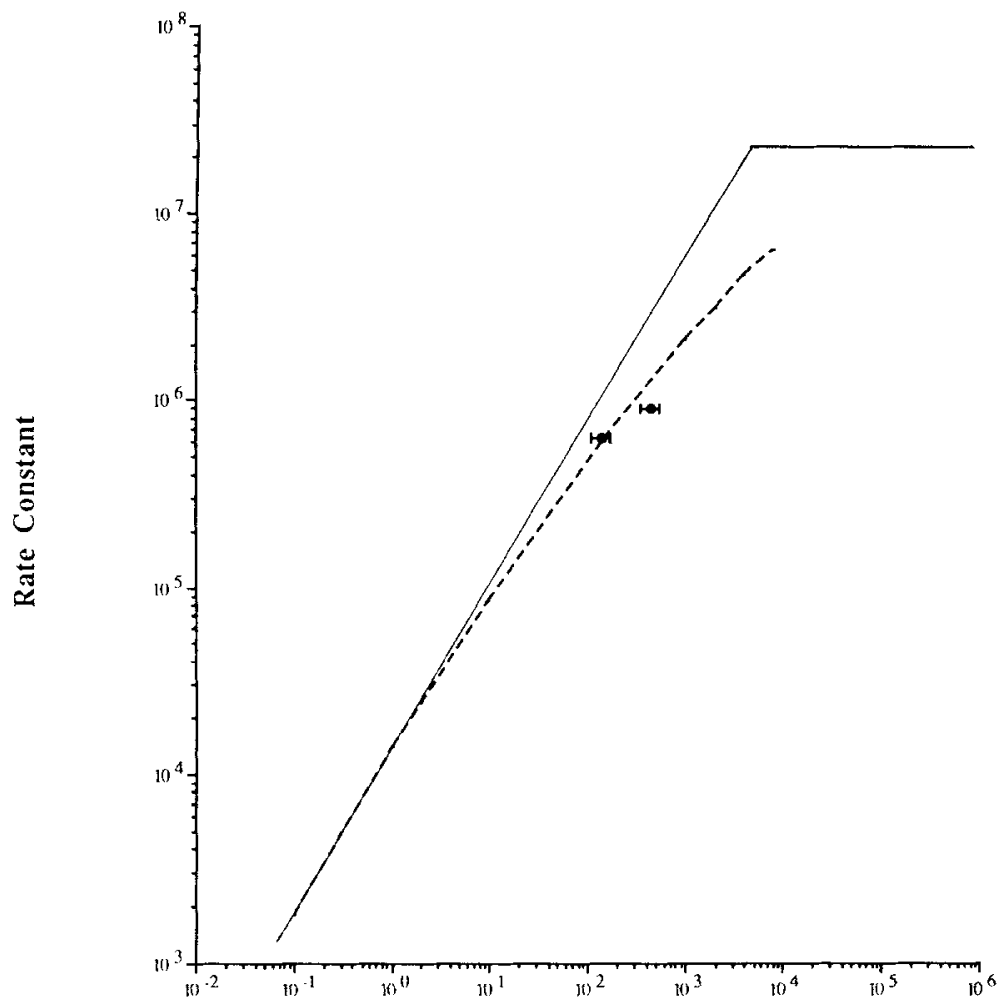

Pressure (torr)

Figure 5. Fall-off for the cyclohexene decompositions at $T=1750 \mathrm{~K}$. The two solid lines are the limiting low pressure and high pressure rate constants. The broken line is the calculated fall-off curve for $\langle\Delta E\rangle_{d}=650 \mathrm{~cm}^{-1}$ with exponential model. The points are from ref. [9]; the bars show the experimental pressure ranges.

time when the effective rate constant has relaxed to within $1 / e$ of $k_{\text {uni }}$.

The incubation times obtained in our calculations are presented in Figure 7, and they show very little sensitivity to temperature, or to collision transition probability model. The incubation time depends almost exclusively on $\langle\Delta E\rangle_{d}$. The magnitudes of these calculated incubation times are similar to, but somewhat greater than the $0.2 \mu \mathrm{s}$ "delay" reported by $\mathrm{KS}$ in their $c-\mathrm{C}_{6} \mathrm{H}_{10}$ shock tube studies [9]. Note that the data analysis of KS included a correction for "delay" and thus their rate constants are consistent with incubation. A slight change in the magnitude of the delay will have negligible effect on the derived rate constants, and Kiefer has indicated that the observed "delay" is probably uncertain by at least a factor of two [21].

Although the simulated incubation times show good agreement with the measurements, various sources of uncertainty in the calculations were investigated. One potential source of uncertainty lies in the high pressure 


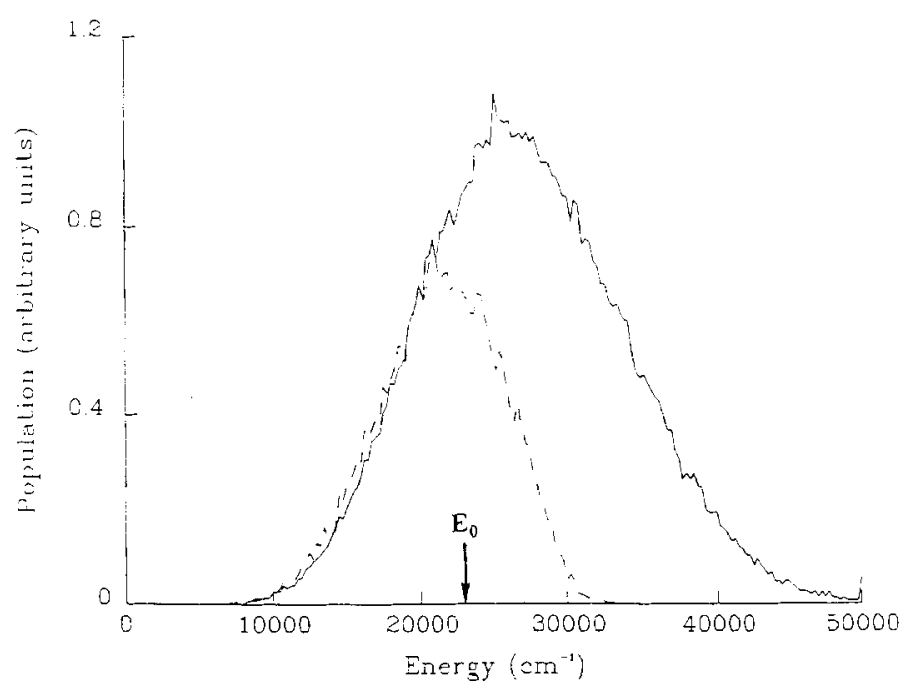

Figure 6. Calculated steady state population of $\mathrm{C}_{6} \mathrm{H}_{10}$, at $T=1750 \mathrm{~K}$. Solid line: $P=8000$ torr; Broken line: $P=0.1$ torr. $\mathrm{E}_{0}=22990 \mathrm{~cm}^{-1}$.

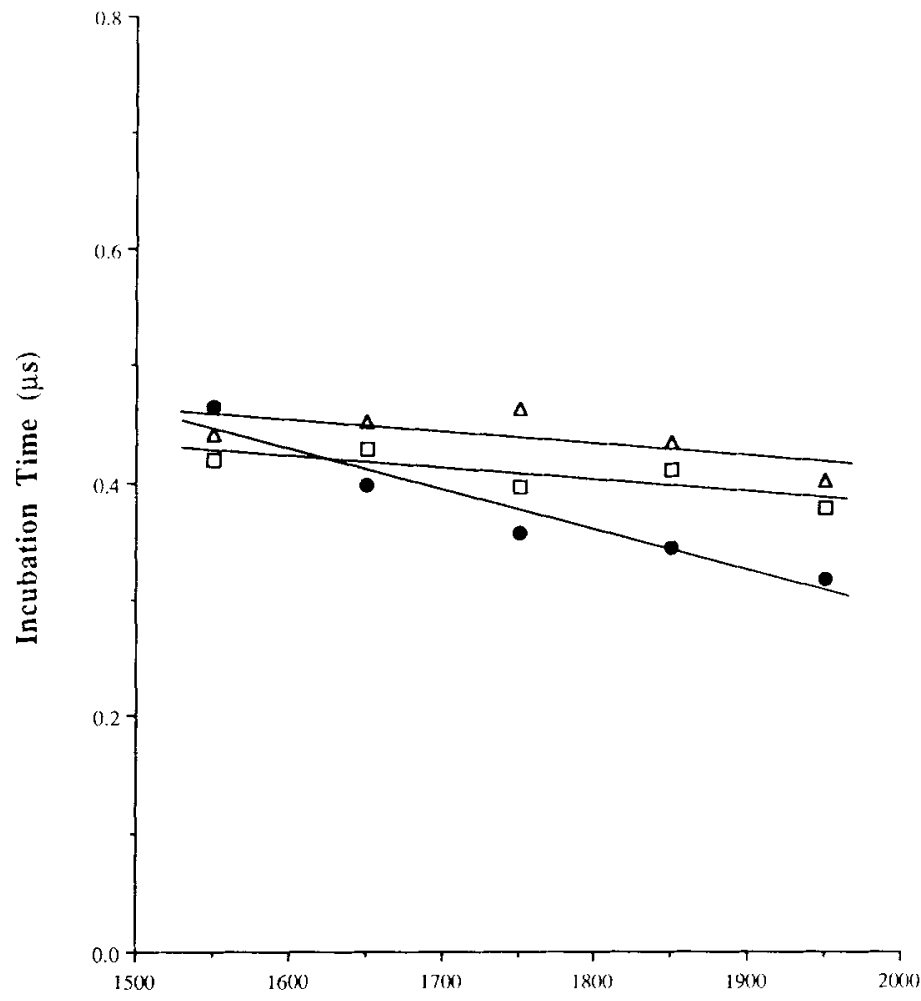

Temperature $(\mathbf{K})$

Figure 7. Incubation time obtained from the simulations with $P=150$ torr. Solid circle: exponential model; Triangle: reverse exponential model; Square: box model. 
rate constant parameters: $A_{\curvearrowright}$ and $E_{\star}$. Calculated $k(E)$ 's using parameters from several different experimental studies agree reasonably well. Nevertheless, as a test, we used the $A_{\infty}$ and $E_{\infty}$ data from Barnard and Parrott [10] to calculate $k(E)$ 's in simulations of the data at $1750 \mathrm{~K}$. We found that a lower $\langle\Delta E\rangle_{d}$ is required to fit the data, but the corresponding incubation time is about $0.3 \mu \mathrm{s}$, not greatly different from the standard simulations and experimental values.

Another source of uncertainty is apparent from Figure 7, which shows scatter in the incubation time data obtained from least squares fitting of the calculated reaction yield data. Both statistical error in the Monte Carlo Master Equation simulations and the error in the least squares fitting contribute to this scatter. Based on the statistical error in the Monte Carlo solution of the Master Equation and on the standard deviations of the least squares fits, this uncertainty is estimated to be less than ca. $0.05 \mu \mathrm{s}$, however.

Uncertainties in the incubation time may also be contributed by the possible energy dependence of $\langle\Delta E\rangle_{d}$. In the calculations to test for this effect, $\langle\Delta E\rangle_{d}$ was assumed to depend on energy according to the expression:

$$
\langle\Delta E(E)\rangle_{d}=g+b E
$$

where $g$ and $b$ are parameters. In order to simplify the description of the energy dependence in the $\langle\Delta E\rangle_{d}$ 's used, we have defined a quantity $f\left(E_{0}\right)$, the degree of energy dependence in $\langle\Delta E\rangle_{d}$ at $E_{0}$, by the following expression:

$$
f\left(E_{0}\right)=\frac{b E_{0}}{g+b E_{0}}
$$

where $E_{0}$ is the critical energy for the $c-\mathrm{C}_{6} \mathrm{H}_{10}$ decomposition $\left(E_{0}=\right.$ $22990 \mathrm{~cm}^{-1}$ ).

Simulations were carried out for cyclohexene at $1750 \mathrm{~K}$ with the Exponential Model, using $\langle\Delta E\rangle_{d}$ 's with different degrees of energy dependence. As shown in Figure 8, the greater the energy dependence, the longer the incubation time. Note that the increase of incubation time is small for low values of $f\left(E_{0}\right)$. Experimental studies and Master Equation simulations of the deactivation of excited azulene by argon indicated that $f(22900) \approx$ 0.6 [12]. If the same energy dependence is assumed for the $c-\mathrm{C}_{6} \mathrm{H}_{10}+\mathrm{Kr}$ system, the incubation time would be about $0.5 \mu \mathrm{s}$, which is probably not easily distinguished experimentally from the ca. $0.2 \mu$ s reported by KS. For comparisons among cases at different temperatures, we have also calculated the second order incubation rate constants, which is defined by: $k=\left\{\tau\left[\mathbf{K}_{r}\right]\right\}^{-1}$, where $\tau$ is the incubation time. The results are shown in Figure 9. A temperature dependence of $T^{-1}$ is obtained, in agreement with Dove and Troe's analysis for $\mathrm{N}_{2} \mathrm{O}$ [6].

At a given temperature, the incubation time can be affected by pressure changes in two ways: (1) changes in collision frequency and (2) changes in the steady-state population distribution. The population distribution at infinite pressure is just the canonical distribution, but at low pressure the population is depleted at energies above the reaction threshold, as mentioned above and shown in Figure 6 . At low pressures, the average energy of reacting molecules $\langle E(T, M)\rangle$ depends on collision frequency and is always 


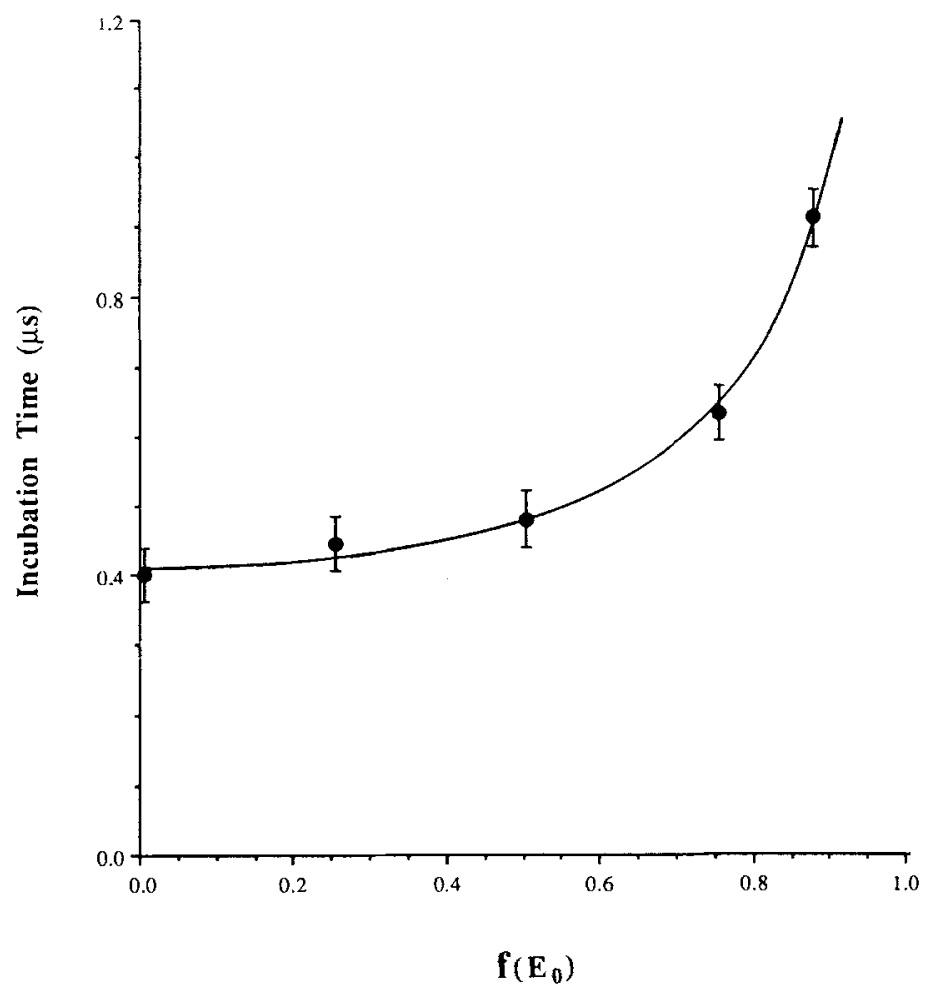

Figure 8. Incubation time as a function of degree of energy dependence in $\langle\Delta E\rangle_{d}$ (defined in text), for the exponential model at $T=1750 \mathrm{~K}$ and $P=150$ torr (see text for details).

lower than the average energy $\langle E(T)\rangle_{T}$ of reacting molecules in a canonical distribution. At the low pressure limit for weak colliders, $\langle E(T, M)\rangle$ is equal to $E_{0}$, the critical energy for reaction. Thus, fewer collisions are needed to reach $\langle E(T, M)\rangle$ than are needed to reach $\langle E(T)\rangle_{T}$. The number of collisions corresponding to the incubation time is $N_{\tau}=Z_{L J}[M]_{\tau}$, which is presented in Figure 10 as a function of the ratio $\langle E(T, M)\rangle /\langle\Delta E\rangle_{d}$. It is clear from this figure that both $N_{\tau}$ and the ratio $\langle E(T, M)\rangle /\langle\Delta E\rangle_{d}$ increase with pressure.

Although fewer collisions are needed to reach $\langle E(T, M)\rangle$ at lower pressure, the incubation time $\tau$ is longer because of the lower collision frequency. In laser schlieren experiments, shock front-laser beam interference is about the same for all pressures [9], and thus it may be possible to measure directly the incubation delay at low pressures, if adequate signals can be observed. Stronger signals may be obtainable by using collider gases with large molar refractivities, such as xenon.

The model dependence of $\langle\Delta E\rangle_{d}$ limits its usefulness as a measure of the energy transfer process. Other measures of energy transfer are the microcanonical quantities such as $\langle\Delta E\rangle$ and $\left\langle\Delta E^{2}\right\rangle^{1 / 2}$, which were calculated at the reaction critical energy for all the temperatures and models, and the results are plotted in Fig. 11(a) and (b). Inspection of the figure shows that 


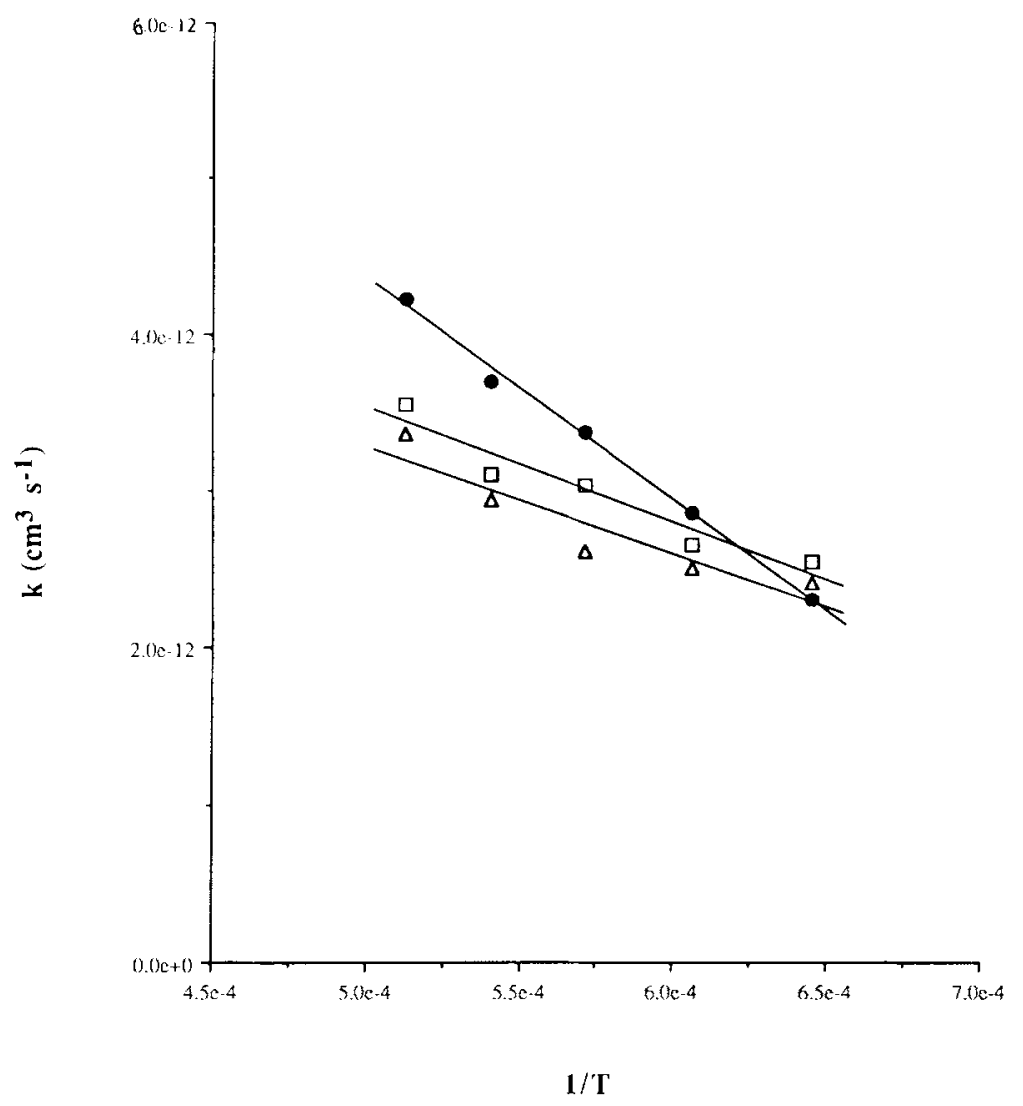

Figure 9. Second order incubation rate constants (as defined in text) as a function of emperature. Solid circle: exponential model; Triangle: reverse exponential model; Square: box model.

$\langle\Delta E\rangle$ is essentially independent of model and but strongly temperaturedependent (unlike $\langle\Delta E\rangle_{d}$ ) and changes sign near $T \approx 1625 \mathrm{~K}$, where the steady-state (at 150 torr) thermal vibrational energy is equal to the critical energy. It has been shown [33,34] that the collisional efficiency for unimolecular reactions is directly related to the second moment $\left\langle\Delta E^{2}\right\rangle$, and it is clear from Figure 11(b) that this quantity has very little dependence on temperature and transition probability model, for the models tested (other models may exhibit a stronger temperature dependence). This characteristic makes it more suitable for estimating collision efficiencies than $\langle\Delta E\rangle_{d}$ and $\langle\Delta E\rangle$, if appropriate correlations among experimental data can be identified.

The large temperature dependence in the microcannonical $\langle\Delta E\rangle$ is shown very clearly in Figure 11(a), where $\langle\Delta E\rangle=0$ at the temperature where the thermal energy of the molecule equals the critical energy $E_{0}$ of the reaction, and becomes positive at higher temperatures [13a]. In KS's simulation of this system [9], they used a constant value, $\langle\Delta E\rangle=-73 \mathrm{~cm}^{-1}$, for both high and low temperature cases, while a constant $\left\langle\Delta E_{d}\right\rangle\left(=550 \mathrm{~cm}^{-1}\right)$ can only simulate the low temperature cases. As pointed out by Tardy and 


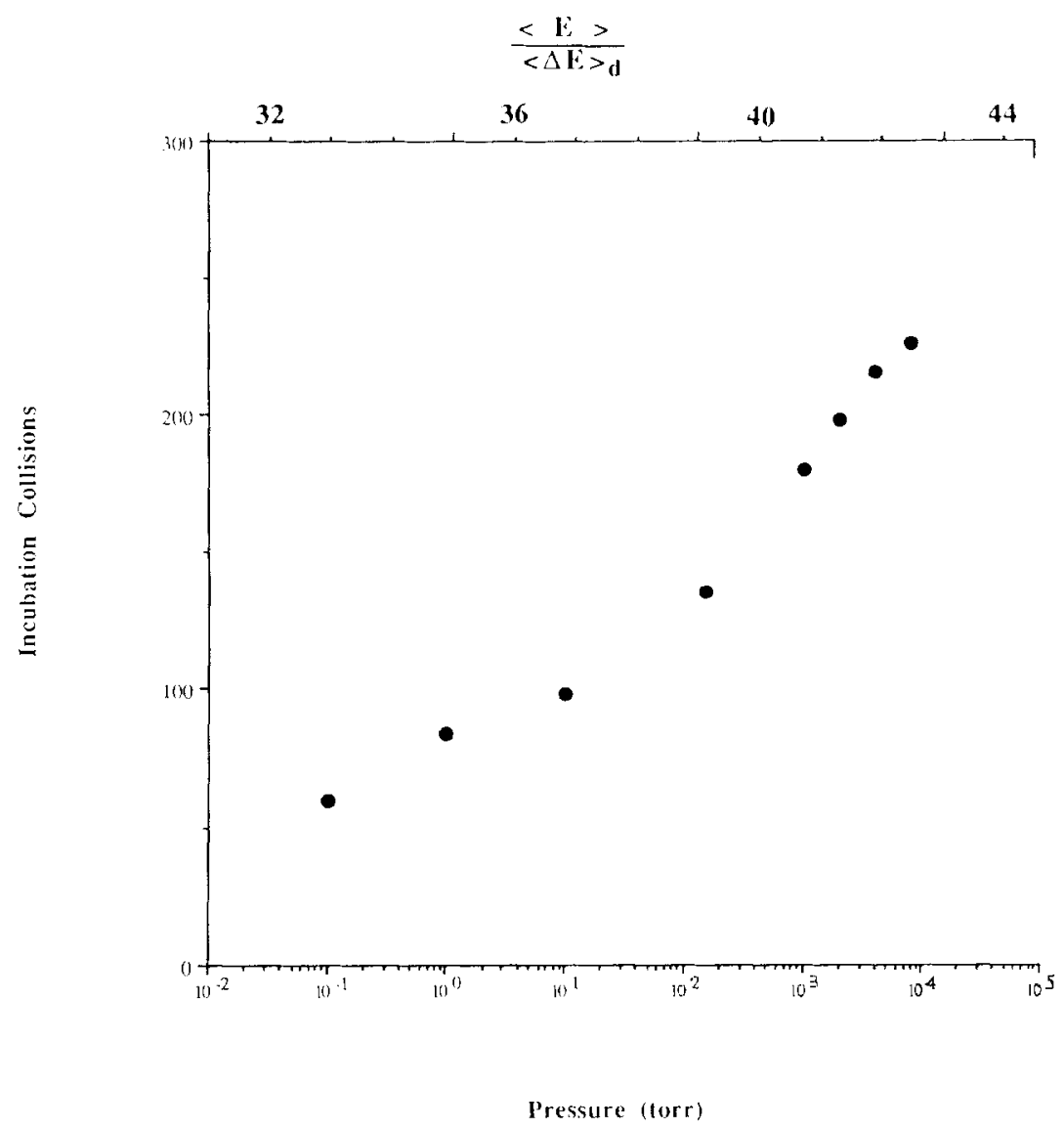

Figure 10. Number of incubation collisions as a function of pressure and $\langle E\rangle /\langle\Delta E\rangle_{d}$, at $T=1750 \mathrm{~K},\langle\Delta E\rangle_{d}=650 \mathrm{~cm}^{-1}$ (exponential model).

Rabinovitch [24], this may be only a fortuitous cancellation of various opposing effects. Tardy and Rabinovich analyzed both the higher- and lower-pressure data of KS and suggested [24] that the collision transfer model may change in this system at high temperatures. We have not considered this possibility in the present work, but it is unlikely to affect our major conclusions.

\section{Conclusions}

Master equation simulations have been used to examine incubation in the decomposition of $\mathrm{C}_{6} \mathrm{H}_{10}$ in shock tube experiments. The calculated incubation time is similar to the "delay" observed by KS in their experiments. This agreement indicates that the experimental "delay" is likely due to incubation. At lower pressures, the incubation times will be longer, suggesting that shock tube schlieren observations of the incubation time might be practical at lower pressures. Signal strengths may be augmented by using collider gases, such as xenon, with higher refractivities. 


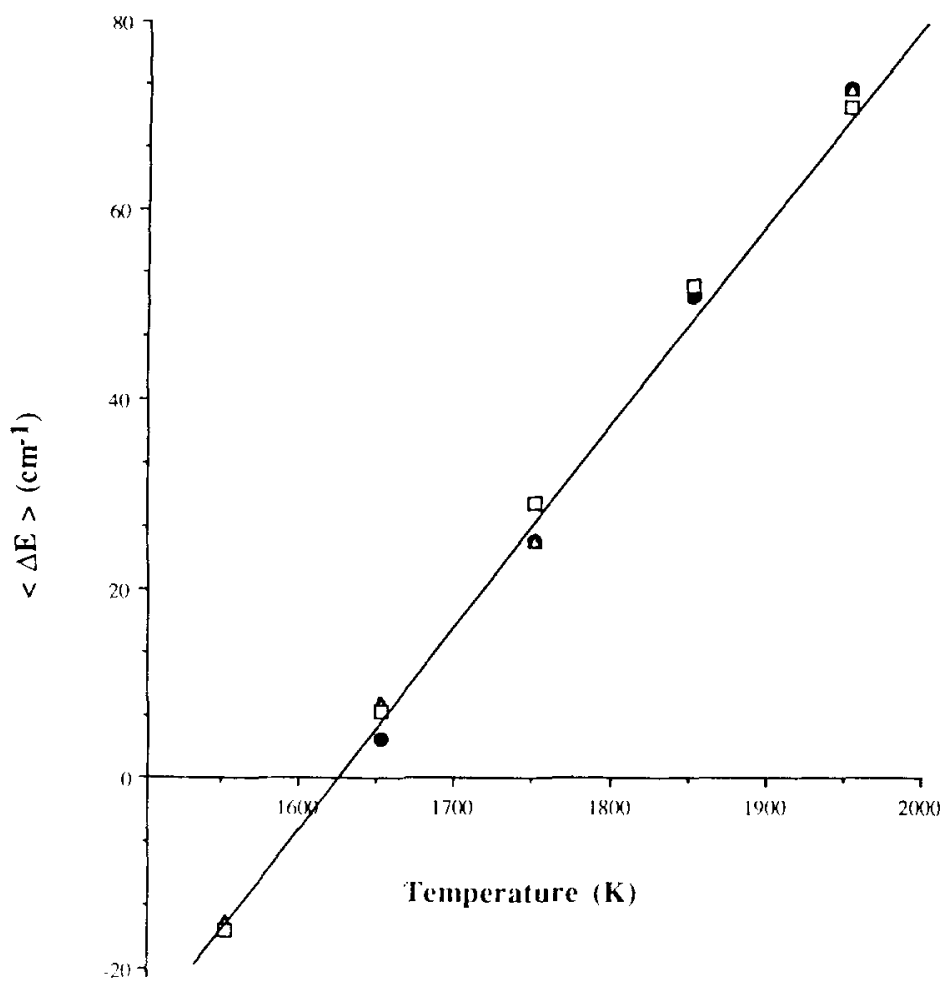

(a)

Figure 11. (a) microcannonical $\langle\Delta E\rangle$ calculated by Eq. (9) (see text). Solid circle: exponential model; Triangle: reverse exponential model; Square: box model. The solid line is the least square fit. (b) microcannonical $\left\langle\Delta E^{2}\right\rangle^{1 / 2}$ calculated by Eq. (10) (see text). Symbols same as in (a).

It was found that the incubation time does not depend strongly on the transition probability model assumed, but it provides a relatively direct measure of $\langle\Delta E\rangle_{d}$. A comparison of $\langle\Delta E\rangle_{d},\langle\Delta E\rangle$, and $\left\langle\Delta E^{2}\right\rangle^{1 / 2}$ shows that the last quantity is almost independent of temperature and assumed transition probability model, unlike the first two, indicating that it may provide a very useful measure of energy transfer.

\section{Acknowledgment}

The authors thank John H. Kiefer for helpful discussions, and W. Forst, I. Oref, D.C. Tardy, and B.S. Rabinovitch for preprints. This work is supported by the Department of Energy (Office of Basic Energy Sciences).

\section{Bibliography}

[1] J. T. Yardley, Introduction to Molecular Energy Transfer, Academic Press, New York, 1980. 


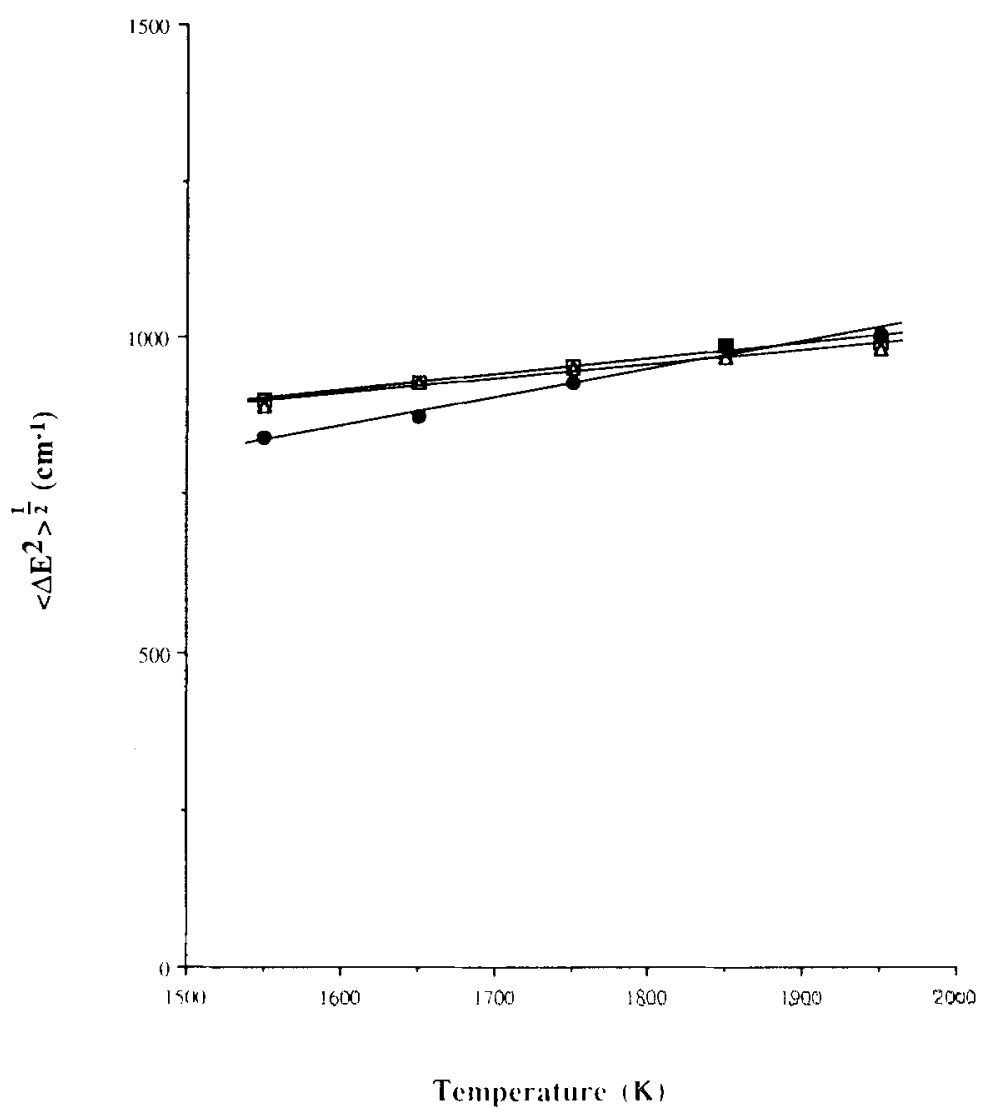

(b)

(Figure 11 continued)

[2] J.E. Dove, W.S. Nip, and H. Teitelbaum, 15th International Symposium on Combustion, The Combustion Institute, Pittsburgh, 1974, p. 903.

[3] H. O. Pritchard, Can. J. Chem., 62, 157 (1984), and references therein.

[4] D.F. Kelley, B.D. Barton, L. Zalotai, and B.S. Rabinovitch, J. Chem. Phys., 71, 538 (1979); R. Arakawa, D.F. Kelley, and B.S. Rabinovitch, J. Chem. Phys., 76, 2384 (1982).

[5] J. R. Barker, Chem. Phys., 77, 301 (1983).

[6] J. E. Dove and J. Troe, Chem. Phys., 35, 1 (1978).

[7] R. J. Malins and D. C. Tardy, Int. J. Chem. Kinet., 11, 1007 (1979).

[8] E. Tzidoni and I. Oref, unpublished work; private communication.

[9] J.H. Kiefer and J. N. Shah, J. Phys. Chem., 91, 3024 (1987).

[10] J. A. Barnard and T. K. Parrott, J. Chem. Soc. Faraday Trans. I, 72, 2404 (1976).

[11] W. Tsang, Int. J. Chem. Kinet., 5, 651 (1973).

[12] (a) J. Shi and J. R. Barker, J. Chem. Phys., 88, 6219 (1988); (b) H. Hippler, B. Otto, and J. Troe, Ber. Bunsenges. Phys. Chem., 93, 428 (1989).

[13] (a) J. R. Barker and R. Golden, J. Phys. Chem., 88, 1012 (1984); (b) M. Heymann, H. Hippler, and J. Troe, J. Chem. Phys., 80, 1853 (1984).

[14] J.H. Kiefer, Shock Waves in Chemistry, A. Lifschitz, Ed., Marcel Dekker, New York, 1981 , p. 219.

[15] J.H. Kiefer, M.Z. Al-Alami, and K. A. Budach, J. Phys. Chem., 86, 808 (1982).

[16] J.E. Dove and H. Teitelbaum, 11th International Symposium on Shock Tubes and Waves, University of Washington Press, Seattle, 1978, p. 474. 
[17] J. H. Kiefer, M. Z. Al-Alami, and J.C. Hajduk, Ap. Optics, 20, 221 (1981).

[18] J. H. Kiefer and A. C. Manson, Rev. Sci. Instr., 52, 1392 (1981).

[19] S. E. Stein and B.S. Rabinovitch, J. Chem. Phys., 58, 2438 (1973).

[20] W. Forst, J. Phys. Chem., 86, 1771 (1982), and reference cited therein.

[21] J. Kiefer, private communication (1988).

[22] W. Forst, J. Phys. Chem., 76, 342 (1972).

[23] J. Troe, J. Ber. Bunsenges. Phys. Chem., 78, 478 (1974).

[24] D.C. Tardy and B.S. Rabinovitch, J. Phys. Chem., to be published.

[25] R. G. Gilbert and K. D. King, Chem. Phys., 49, 367 1980).

[26] T. T. Nguyen, K. D. King, and R. G. Gilbert, J. Phys. Chem., 87, 494 (1983).

[27] R. G. Gilbert, Chem. Phys. Lett., 96, 259 (1983).

[28] J. R. Barker, J. Phys. Chem., 88, 11 (1984).

[29] J.R. Barker and T. Rothem, Chem. Phys., 68, 331 (1982); P. L. Trevor, T. Rothem, and J. R. Barker, Chem. Phys., 68, 341 (1982); J.R. Barker and B. M. Toselli in Photothermal Investigations of Solids and Fluids, Jeffery A. Sell, Ed., Academic, New York, 1989 p. 155.

[30] N. Presser, J. R. Barker, and R. J. Gordon, J. Chem. Phys., 78, 2163 (1983); K. M. Beck, A. Ringwelski, and R.J. Gordon, Chem. Phys. Lett., 121, 529 (1985).

[31] H. Hippler, L. Lindemann, and J. Troe, J. Chem. Phys., 83, 3906 (1985).

[32] E. E. Nikitin, Theory of Thermally Induced Gas Phase Reactions, E. W. Schlag, Ed., Indiana University Press, Bloomington, 1966, p. 136.

[33] W. Forst, J. Phys. Chem.,. 93, 3145 (1989); W. Forst, J. Phys. Chem., 90, 456 (1986).

[34] J. Troe, J. Chem. Phys., 66, 4745 (1977).

[35] However, stronger temperature dependences are found in some thermal unimolecular reaction studies; see ref. [13(a)] for discussion.

Received May 8, 1989

Accepted August 22, 1989 